Review Article

\title{
RNA-binding proteins and their role in the regulation of gene expression in Trypanosoma cruzi and Saccharomyces cerevisiae
}

\author{
Camila Oliveira ${ }^{1}$, Helisson Faoro ${ }^{1}$, Lysangela Ronalte Alves ${ }^{1}$ and Samuel Goldenberg ${ }^{1}$ \\ ${ }^{1}$ Instituto Carlos Chagas, Fiocruz-Paraná, Curitiba, PR, Brazil.
}

\begin{abstract}
RNA-binding proteins (RBPs) have important functions in the regulation of gene expression. RBPs play key roles in post-transcriptional processes in all eukaryotes, such as splicing regulation, mRNA transport and modulation of mRNA translation and decay. RBPs assemble into different mRNA-protein complexes, which form messenger ribonucleoprotein complexes (mRNPs). Gene expression regulation in trypanosomatids occurs mainly at the post-transcriptional level and RBPs play a key role in all processes. However, the functional characterization of RBPs in Trypanosoma cruzi has been impaired due to the lack of reliable reverse genetic manipulation tools. The comparison of RBPs from Saccharomyces cerevisiae and T. cruzi might allow inferring on the function of these proteins based on the information available for the orthologous RNA-binding proteins from the S. cerevisiae model organism. In this review, we discuss the role of some RBPs from $T$. cruzi and their homologues in regulating gene expression in yeast.
\end{abstract}

Keywords: RNA-binding proteins, Trypanosoma cruzi, Saccharomyces cerevisiae, gene expression regulation.

Received: September 28, 2016; Accepted: January 10, 2017.

\section{Introduction}

Gene expression involves several events that occur at the transcriptional and post-transcriptional levels. The transcriptional control of gene expression has been extensively influenced by early work on bacterial transcription. However, in recent years, post-transcriptional events have gained much more attention. The pre-RNA undergoes extensive processing before the mRNA reaches its final destination and RNA-binding proteins (RBPs) associated to the RNA during its life-time play a key role in determining its fate in the cell. (Kishore et al., 2010). The association of proteins with mRNAs is very dynamic and prone to changes according to the environment. Consequently RBPs are involved in the stabilization or destabilization of mRNAs in response to stress or extracellular signals (Alves and Goldenberg, 2016).

The availability of high-throughput analysis techniques, such as proteomics, has enabled the characterization of several RBPs. Nevertheless, the RBP network assembly and the mechanism of the RNA regulon are still poorly explored, and further work is required to determine the identity of all of the proteins and their respective roles in post-transcriptional events (Lunde et al., 2007).

Send correspondence to Lysangela Ronalte Alves or Samuel Goldenberg. Instituto Carlos Chagas, Fiocruz-Paraná, Rua Professor Algacyr Munhoz Mader 3775, Cidade Industrial de Curitiba, 81350-010 Curitiba, PR, Brazil. E-mail: lysalves@fiocruz.br; sgoldenb@fiocruz.br.
RBPs have one or multiple RNA-binding protein domains. The following are the best characterized RNAbinding domains: RNA Recognition Motif (RRM), Khomology domain (KH), RGG (Arg-Gly-Gly) box, zinc finger, double stranded RNA-binding domain (dsRBD), Pumilio/PUF domain and Piwi/Argonaute/Zwille (PAZ) domain (Finn et al., 2010).

The RRM is the most abundant domain and also the most studied in RBPs (Afroz et al., 2015). The information obtained from genome sequencing studies shows that RRM-containing proteins are present in all forms of life (Mari et al., 2005). RRMs typically comprise approximately 90 amino acids and consist of four antiparallel $\beta$-strands (eventually they can have one or two short additional strands), which form a $\beta$-sheet that is packed against two $\alpha$-helices, adopting the typical $\beta 1 \alpha 1 \beta 2 \beta 3 \alpha 2 \beta 4$ conformation. The $\beta 3$ and $\beta 1$ strands of the RRM contain the RNP1 and RNP2 signature sequences, respectively (Cléry and Allain, 2012). Additionally, two or more RRMs can be combined in the same molecule to recognize longer stretches of RNA, with increased sequence affinity and specificity (Cléry and Allain, 2012).

The hnRNP K-homology (KH) domain comprises three $\alpha$-helices around the surface of a central antiparallel $\beta$-sheet. Eukaryotic type I and prokaryotic type II KH domains share a minimal $\beta \alpha \alpha \beta$ core, with two additional $\alpha$ and $\beta$ elements positioned either in C-terminal (type I, eukaryotes) or N-terminal (type II, prokaryotes) orientation 
to this core motif (Grishin, 2001). This structure directs four nucleic acid bases towards a groove inside the protein structure where hydrophobic interactions and a network of main chain and side chain hydrogen bonds mediate nucleobase recognition. So far, protein domains with a classical $\mathrm{KH}$ fold but lacking a conserved GxxG motif have shown no nucleic acid-binding activity, although they interact with other nucleic acid binding domains and can modulate their RNA binding activity (Valverde et al., 2008).

The RGG motif is an evolutionarily conserved sequence. In addition to the arginine and glycine repeats, aromatic residues are frequently observed in-between these sequences, and these residues may contribute to hydrophobic stacking within RNA bases. RGG/RG motives include RGG and RG repeats of varied lengths interspersed with spacers of different amino acids (Corley and Gready, 2008), and predicting the spacing that defines a functional $\mathrm{RGG} / \mathrm{RG}$ motive is difficult. The structure of the RGG/RG has not been clearly defined due to its low sequence complexity.

Classical $\mathrm{C} 2 \mathrm{H} 2$ 'zinc finger' proteins were identified as modular nucleic acid recognition elements, with two cysteine and two histidine residues that coordinate a zinc ion. Although mostly noted for their role as DNA-binding transcription factors, $\mathrm{C} 2 \mathrm{H} 2$ zinc fingers were identified in the transcription factor IIIA (TFIIIA) (Vincent, 1986). TFIIIA contains nine $\mathrm{C} 2 \mathrm{H} 2$ zinc fingers, which are used to recognize RNA and DNA targets. The zinc finger folds into a small domain comprising two $\beta$ strands followed by one $\alpha$ helix. More recently, the $\mathrm{C} 2 \mathrm{H} 2$ class of zinc finger protein has been shown to bind preferentially to RNA targets. These zinc fingers are characterized by three cysteine residues and one histidine residue that coordinate the zinc ion and form the Cys-X7-8-Cys-X5-Cys-X3-His sequence (Hall, 2005).

The dsRBD is a conserved protein domain of approximately 65-70 amino acids which binds to double-stranded or highly structured RNAs (Finn et al., 2010). The dsRBD was first recognized as a conserved protein domain based on the similarities between Drosophila Staufen, human TAR-RNA binding protein (TRBP) and Xenopus laevis RNA-binding protein A (XlrbpA). The central function of dsRBDs is to bind to dsRNA regions, which is primarily achieved by recognizing specific RNA shapes. In addition to this major function, dsRBDs with protein-protein interaction properties have been reported to participate in the regulation of protein subcellular localization, suggesting that the participation of dsRBDs in nucleocytoplasmic trafficking is likely to represent a widespread auxiliary function of this type of RNA-binding domain (Banerjee and Barraud, 2014).

Pumilio is a family of sequence-specific RNAbinding proteins that regulate translation of the mRNA targets and also appear to interact with mRNA regulatory sys- tems (Edwards, 2015). RNA recognition by Pumilio occurs through the PUF domain, named after its members Pumilio and FBF. Full-length Pumilio is a relatively large protein (156 kDa in Drosophila); however, only a fraction of the Pumilio protein (a $37 \mathrm{kDa}$ fragment close to the protein $\mathrm{C}$-terminus) is required for RNA binding, translational repression, and recruitment of other proteins. The PUF domain contains multiple tandem repeats of 35-39 amino acids which recognize specific RNA bases (Abbasi et al., 2011).

The PAZ domain is found in Dicer and Argonaute proteins, two protein families with key roles in RNAi mechanisms. The PAZ domain consists of two subdomains, one of which displays OB-like folding (oligonucleotide/oligosaccharide binding). Hence, the PAZ motif might bind to single-stranded nucleic acids (Yan et al., 2003). Crystallographic studies combined with biochemical approaches showed that the PAZ domain binds to ssRNAs with low affinity in a sequence-independent manner. A remarkable feature of the PAZ domain is that it can recognize the 3'-ends of ssRNAs. Both miRNAs and distinct types of small interfering RNAs (siRNA) are processed by the sequential action of RNase III enzymes (Drosha and Dicer in mammals, or Dicer alone in yeast and plants), which characteristically leave two 3'-overhangs on the processed products (Hutvagner and Simard, 2008).

\section{RNA-binding proteins in Trypanosomatids}

The regulation of gene expression in trypanosomatids occurs mainly by post-transcriptional mechanisms. These protozoans present several peculiarities, such as a less condensed chromatin structure, polycistronic transcription, a trans-splicing mechanism, and the absence of canonical RNA polymerase II promoters. Genome analysis of the TriTryp database (containing genome sequences of the pathogenic T. cruzi, Leishmania major and Trypanosoma brucei) shows several RNA-binding proteins. Nonetheless, a comprehensive characterization of RNA-protein interactions remains elusive (Clayton and Shapira, 2007).

In 2005, De Gaudenzi and co-workers described approximately 80 proteins with RRM domains in $T$. cruzi, but few were functionally characterized (Table 1) (De Gaudenzi et al., 2005). Another comprehensive study was conducted to characterize ribonucleoprotein complexes (mRNPs) in T. cruzi (Alves et al., 2010). In this study, several RBPs were identified by proteomics, using polysomal and polysome-free fractions of exponentially growing epimastigotes and epimastigotes under conditions of nutritional stress.

The life cycle of $T$. cruzi involves two hosts (triatomine insects and mammals) and comprises four morphological stages, two replicative (epimastigotes in the insects and amastigotes in the mammalian cells) and two infective forms (metacyclic trypomastigotes in the insects and 
Table 1 - RNA binding proteins characterized in Trypanosoma cruzi.

\begin{tabular}{lccc}
\hline Protein & Function & Ref. & Domain \\
\hline SR62 & mRNA processing/stability & Názer et al. (2011) & SR-related \\
ZC3H39 & Regulator of a specific subset of mRNAs & Alves et al., 2014 & CCCH \\
UBP1 & mRNA destabilizing factor & D'Orso and Frasch (2002) & RRM \\
UBP2 & mRNA destabilizing factor & D'Orso and Frasch (2002) & RRM \\
PUF6 & mRNA destabilizing factor & Dallagiovanna et al. (2008) & Pumilio \\
ZFP1 & Involved in differentiation & Mörking et al. (2004) & CCCH \\
ZFP2 & Involved in differentiation & Mörking et al. (2004, 2012) & CCCH \\
ZFP3 & Involved in differentiation, translation regulator & Mörking et al. (2004) & CCCH \\
RBP40 & Regulator of a specific subset of mRNAs & Guerra-Slompo et al. (2012) & RRM \\
RBP19 & Involved in differentiation & Pérez-Díaz et al. (2012, 2013) & RRM \\
DRBD4/PTB2 & Involved regulation of splicing & De Gaudenzi et al., (2016) & RRM \\
PABP1 & Involved in translation & Batista et al. (1994) & RRM \\
\hline
\end{tabular}

bloodstream trypomastigotes in mammals). The epimastigotes differentiate in the midgut of the insect host and become metacyclic trypomastigotes, which are released in the excreta when the triatomine feeds on blood. The parasites penetrate the body of the mammalian host through the damaged skin or mucosa and invade different cell types. Within the cells, the parasites differentiate into amastigotes)De Souza, 2002).

\section{RNAi in T. cruzi and yeast}

The canonical RNAi machinery comprises three main components: Dicer, Argonaute, and RNA-dependent RNA polymerase. Argonaute proteins contain two conserved domains, the PAZ and Piwi domains. These proteins are components of the RNA-induced silencing complex (RISC) (Liu et al., 2004). Fungi, such as Ascomycetes, Basidiomycetyes, and Zygomycetes present the RNA silencing components in the genome, while few ascomycete and basidiomycete fungi apparently lost these components (Nakayashiki et al., 2006).

Saccharomyces cerevisiae, T.a cruzi, L. major and Plasmodium falciparum do not have the RNAi machinery, which seems to have been lost or excessively simplified. However, an ORF encoding for an AGO/PIWI protein expressed in all stages of the life cycle of $T$. cruzi was recently described (Garcia-Silva et al., 2010). The results showed that the TcPIWI-tryp is a canonical Argonaute in its domain architecture (Garcia-Silva et al., 2010). Moreover, it was shown that the most represented sRNAs interacting with TcPIWI-tryp derived from rRNAs, which corresponded to known miRNAs of higher eukaryotes, indicating a possible evolutionary pathway of known canonical sncRNAs from structural RNAs (Garcia-Silva et al., 2014).

\section{RBPs with RRM domain in T. cruzi}

Some RBPs play an important role during the differentiation of the parasite by regulating the expression of spe- cific transcripts. TcUBP-1 recognizes the AU-rich instability element located in the 3'-untranslated region (UTR) of mucin SMUG mRNAs (D'Orso and Frasch, 2002). TcUBP-2 binds to poly(U)-RNA and is differentially expressed during parasite development. Both proteins interact in the same complex and are implicated in controlling $T$. cruzi SMUG mucin mRNA levels. In addition, they are located preferentially in the polysomal fraction (D'Orso and Frasch, 2002).

TcRBP40 binds to AG-rich regions in the 3'-UTR of target mRNAs. Microarray data indicate that this protein binds to mRNAs encoding various transmembrane proteins. The TcRBP40 protein location varies throughout the parasite's life cycle. In the epimastigote stage It is localized in reservosomes, which are trypanosomatid organelles associated to protein and lipid storage, and in amastigotes and trypomastigotes it is dispersed in the cytoplasm, suggesting a potential gene regulatory function (Guerra-Slompo et al., 2012).

TcRBP19 is differentially expressed during the life cycle of $T$. cruzi and is not detected only in the amastigote stage. Regulation of TcRBP19 is mediated by the 3'-UTR region, and the overexpression of TcRBP19 affects the $T$. cruzi life cycle and ability for infection (Pérez-Díaz et al., 2012, 2013). Recently, De Gaudenzi et al. (2016), showed that TcDRBD4/PTB2 is an essential multifunctional RBP, involved in regulation of splicing, preventing trans-splicing and decreasing both UBP1 and UBP2 proteins expression

TcPABP1 was first characterized in 1994 by Batista et al. (1994), showing that this protein has been conserved throughout eukaryotic evolution. This Poly (A) binding protein has been more extensively described in $T$. brucei than in T. cruzi. PABP1 and PABP2 are localized in different sets of granules in response to inhibition of either translation or trans-splicing. PABP2 co-localized with the marker DHH1 into RNP granules, which are similar to P-bodies, and in nuclear periphery granules, whereas 
PABP1 is localized in heat shock induced stress granules (Kramer et al., 2013).

\section{RBPs with PUF domains in T. cruzi}

The PUF family of RNA-binding proteins regulates their target mRNAs by binding to their 3'-UTR. In T. cruzi, the TcPUF6 protein is involved in the degradation of specific mRNAs, especially those that are upregulated in the infective trypomastigote form (Dallagiovanna et al., 2008).

\section{RBPs with the $\mathrm{CCCH}$ zinc finger domain in $T$. cruzi}

The T. cruzi proteins TcZFP1 and TcZFP2 have been characterized and contain the $\mathrm{C} 2 \mathrm{H} 2$ domain. TcZFP1 binds specifically to oligoribonucleotides containing cytosinerich sequences. This type of repetitive sequence is present in untranslated regions of many mRNAs in trypanosomatids (Mörking et al., 2004). Ribonomic analysis showed that the targets of the protein TcZFP2 are associated with parasite-host interactions, for which expression is downregulated in the replicative forms, indicating that TcZFP2 protein might act by destabilizing its targets (Mörking et al., 2012). The protein TcZC3H39 sequesters highly expressed mRNAs and their associated ribosomes, slowing translation under stress conditions. In addition, the transcript content is changed in normal and stressful conditions, and most of its targets code for cytochrome c oxidase enzymes (COX) and ribosomal proteins, presenting evidence for the RNA regulon theory (Alves et al., 2014).

\section{Other RBP domains in T. cruzi}

Some RBPs involved in mRNA metabolism can be relocalized to the nucleolus in T. cruzi as a specific stress response. TcSR62 is an RBP that belongs to the SR-related protein family, which is implicated in several functions related to mRNA metabolism. TcSR62 is involved in mRNA processing/stability, since its overexpression in T. brucei affects the mRNA trans-splicing process and leads to a decreased abundance of several mRNAs (Názer et al., 2011).

When mRNAs are not translated, they are compartmentalized into cytoplasmic structures named RNA granules. These RNA granules comprise the 'processing bodies' ('P-bodies') and the stress granules. Several RBPs have been implicated in the assembly and/or maintenance of these structures. TcDHH1, a putative DEAD-box RNA helicase, is involved in multiple RNA-related processes in various eukaryotes and accumulates in stress granules and P-bodies of yeast, animal cells and T. brucei (Kramer et al., 2010). In T. cruzi, DHH1 is present in heavy protein complexes, which are not associated with the polysome complexes, and is located diffusely in the cytoplasm under normal conditions. However, DHH1 forms cytoplasmic granules upon nutritional stress or treatment with drugs that dissociate the polysomes (Holetz et al., 2010).

\section{RNA-binding proteins in yeast}

The RNA-RBP complexes can be identified by RBP immunoaffinity purification (RIP), where the proteins are purified together with the bound RNAs, and the associated RNAs can then be identified. CLIP (cross-linking and immuno-precipitation) is a method that can directly determine the binding sites of RBPs onto mRNA. A substantial number of mRNA-binding proteins from yeast were identified from studies on the mechanisms of biogenesis, localization, translation and degradation of mRNAs (Mitchell et al., 2013).

\section{RBPs with an RRM domain in S. cerevisiae}

RBPs with RRM domains are well characterized in $S$. cerevisiae. This is the case of PABP1 (Poly-A binding protein), which contains four RRM domains (Figure 1), and is found in the cytoplasm, where it is associated with mRNA poly-A tails, stimulating translation initiation and regulating mRNA stability (Amrani et al., 1997).

The second best studied protein in yeast is PUB1, which has three RRMs and can be located both in the nucleus and the cytoplasm, and is associated with poly(U) sequences (Anderson et al., 1993). PUB1 is involved in the stabilization of mRNAs containing ARE ("AU-rich elements"), and it is also involved in the process of nonsense-mediated mRNA decay (NMD) (Ruiz-Echevarría and Peltz, 2000).

The ScPRP24 protein also contains three RRM domains and is involved in the formation and organization of the spliceosome complex (Shannon and Guthrie, 1991). Moreover, the RRM domains 2 and 3 of ScPRP24 stabilize the U6 RNA and allow it to complete the U4/U6 RNA interaction, thereby influencing the association and dissociation of U4 and U6 RNAs with ScPRP24 (Vidaver et al., 1999).

\section{RBPs with PUF domain in S. cerevisiae}

Yeast possesses six PUF proteins (named PUF1-PUF6), and these proteins modulate mRNA stability through association with the 3'-UTR of their target mRNAs. For example, PUF1p activity involves recognition of UGUA sequences and surrounding sequences by PUF proteins. PUF also regulates several mitochondrial proteins, such as PMP1, PMP2, PMP3, and AST1. These mRNAs have been associated with PUF1p and/or PUF2 $p$ and encode membrane-associated proteins involved in proton transport (Ulbricht and Olivas, 2008). PUF3 promotes the deadenylation of Cox17 (Olivas and Parker, 2000), while PUF4 and PUF5 act on the deadenylation and decay of $\mathrm{HO}$, a specific endonuclease that stimulates mating-type switching in budding yeast (Tadauchi et al., 2001). Interestingly, PUF6 (Figure 2) acts on the regulation of Ash1, which represses $\mathrm{HO}$ in cells to block mating-type switching (Gu et al., 2004). 
A

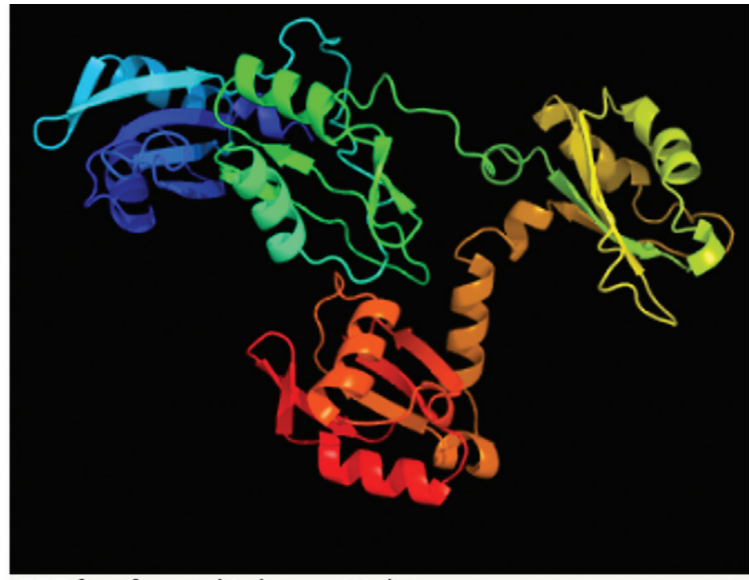

PDB header:rna binding protein/rna

Chain: A: PDB Molecule:u4/u6 snrna-associated-splicing factor prp24;

PDBTitle: core structure of the u6 small nuclear ribonucleoprotein at 1.72 angstrom resolution

\begin{tabular}{l|c|c}
\hline & \multicolumn{2}{c}{ Confidence and coverage } \\
Confidence: & $\mathbf{1 0 0 . 0} \%$ & Coverage: $60 \%$
\end{tabular}

349 residues ( $60 \%$ of your sequence) have been modelled with $100.0 \%$ confidence by the single highest scoring template.
B

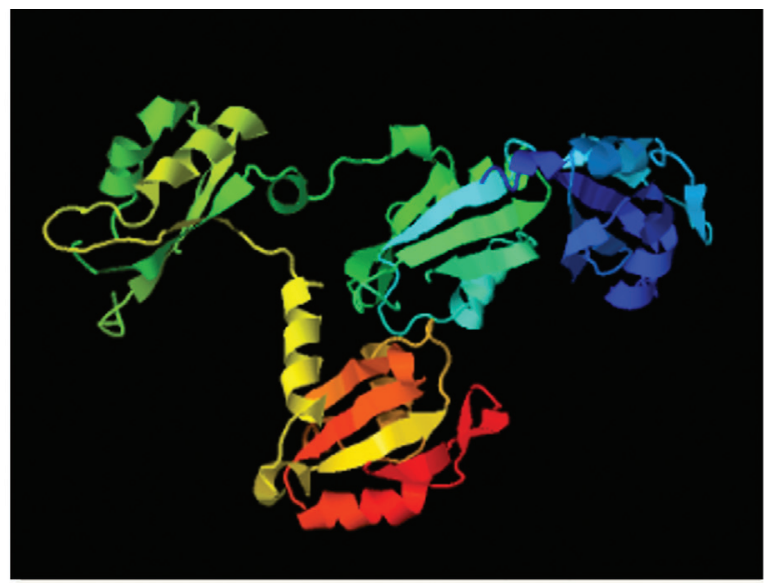

PDB header:rna binding protein/rna

Chain: A: PDB Molecule:u4/u6 snrna-associated-splicing factor prp24;

PDBTitle: core structure of the $\mathrm{u} 6$ small nuclear ribonucleoprotein at 1.72 angstrom resolution

\begin{tabular}{l|c|c}
\hline & & Confidence and coverage \\
Confidence: & $\mathbf{1 0 0 . 0} \%$ & Coverage: $61 \%$
\end{tabular}

345 residues ( $61 \%$ of your sequence) have been modelled with $100.0 \%$ confidence by the single highest scoring template.

Figure 1 - Structural prediction of ScPab1 (A) and TcPabp1 (B) proteins (Phyre2 program).

A

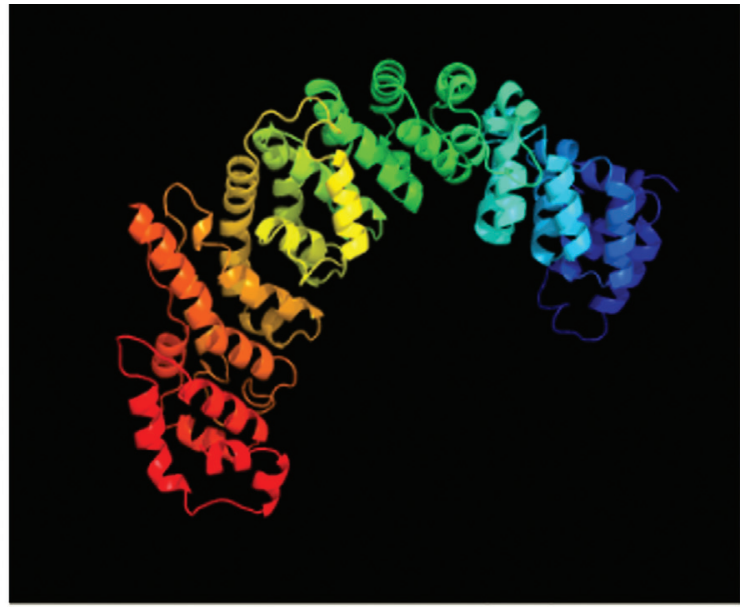

PDB header:dna binding protein, rna binding protein

Chain: A: PDB Molecule:pumilio domain-containing protein kiaa0020;

PDBTitle: crystal structure of human puf-a in complex with dna

\begin{tabular}{l|c|c|c} 
& & Confidence and coverage \\
Confidence: & $\mathbf{1 0 0 . 0 \%}$ & Coverage: $770 \%$
\end{tabular}

504 residues ( $77 \%$ of your sequence) have been modelled with $100.0 \%$ confidence by the single highest scoring template.
B

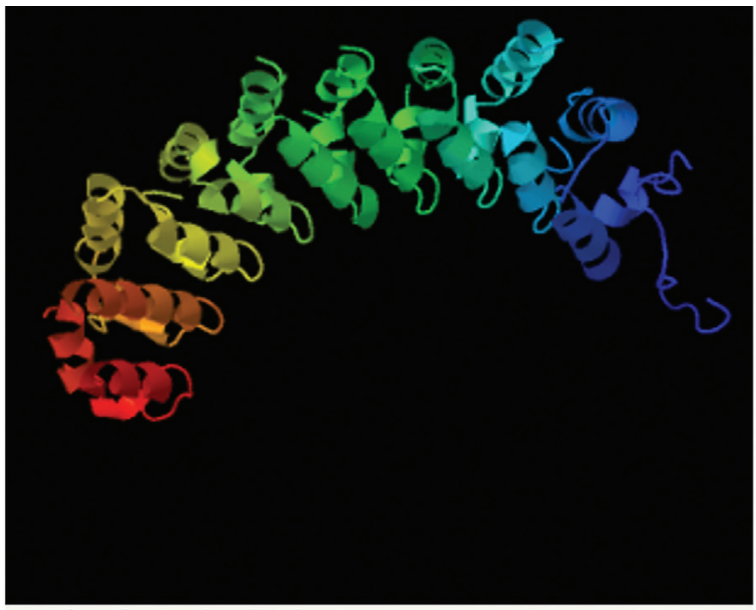

PDB header:rna/rna binding protein

Chain: A: PDB Molecule:fem-3 mrna-binding factor 2; PDBTitle: crystal structure of fbf-2/fem-3 pme complex

Confidence and coverage

Confidence:

$100.0 \%$

Coverage: $41 \%$

335 residues ( $41 \%$ of your sequence) have been modelled with $100.0 \%$ confidence by the single highest scoring template.

Figure 2 - Structural prediction of ScPuf6 (A) and TcPuf6 (B) proteins (Phyre2 program).

RBPs with zinc finger $\mathrm{CCCH}$ domains in $\mathrm{S}$.

\section{cerevisiae}

CTH1 (Figure 3) and CTH2 were first described in yeast. Both proteins can play a role in mRNA activation or degradation of mRNA targets involved in iron homeostasis (Thompson et al., 1996).
Two zinc finger proteins, MSN2 and MSN4, function as transcriptional activators (Estruch and Carlson 1993), and under stress conditions both proteins can activate one or more genes involved in the protective response following different types of stress (Martínez-Pastor et al., 1996). 


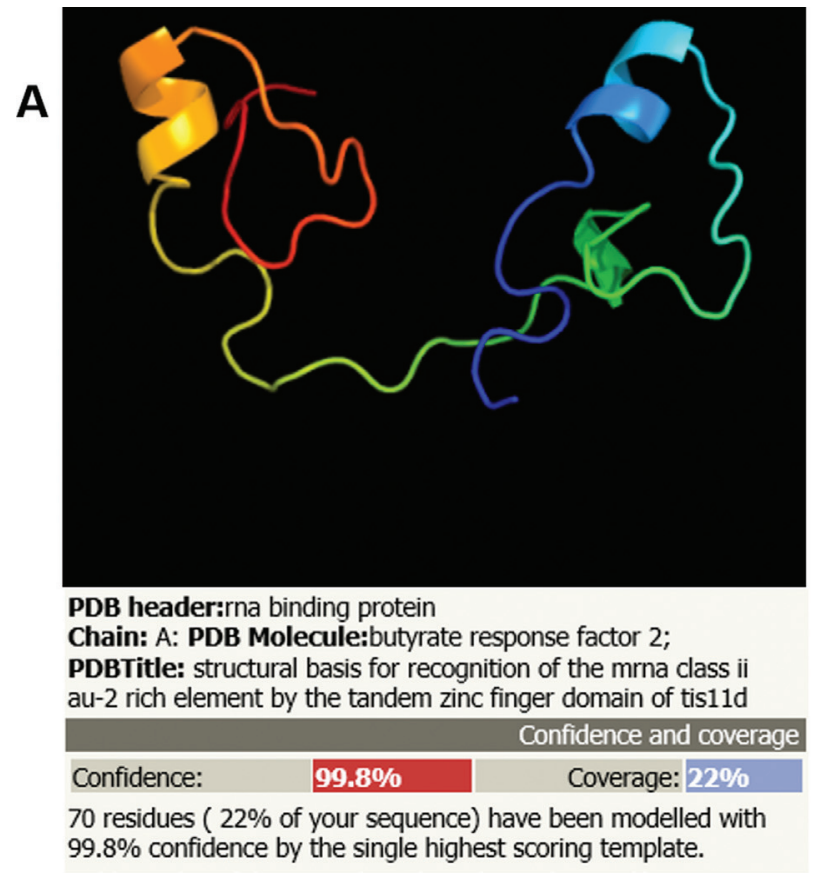

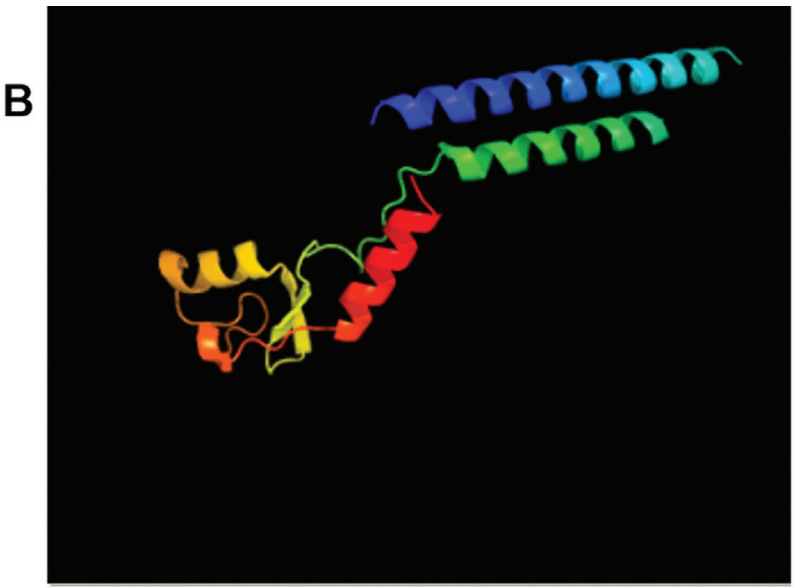

PDB header:chaperone

Chain: A: PDB Molecule:stip1 homology and u-box containing protein 1;

PDBTitle: dimerization and u-box domains of zebrafish c-terminal of hsp702 interacting protein

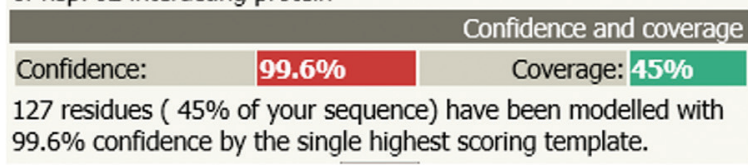

Figure 3 - Structural prediction of ScCth1 (A) and TcZC3h39 (B) proteins (Phyre2 program).

\section{Other RBP domains in S. cerevisiae}

There are many other RBPs that have been characterized. For example, SCP160 is a protein that has 14 repeats of the KH domain (Figure 4) and is associated with polyribosome bound mRNPs (Lang and Fridovich-Keil, 2000). Interestingly, this protein also participates in the formation of P-bodies, since it appears to prevent P-bodies formation under normal conditions (Weidner et al., 2014).

\section{RBPs orthology between T. cruzi and S. cerevisiae}

To investigate if the RBP proteins of $T$. cruzi are present in S. cerevisiae we performed an orthology analysis. The RBP amino acid sequences from T. brucei (De Gaudenzi et al., 2005) were used to identify RBPs in T. cruzi through best reciprocal Blast hit analysis, resulting in 61 proteins with identity ranging from 87.04 to $30.38 \%$. The identified proteins were then compared to all encoded proteins of $S$. cerevisiae genome using the same approach. A total of $20 \mathrm{~T}$. cruzi proteins were found orthologous in $S$. cerevisiae, but the overall identity was lower, ranging from 44.44 to $22.17 \%$ (Table 2). Despite the low identity between T. cruzi and S. cerevisiae proteins, domain analysis showed that the proteins had related RBP domains, suggesting that these proteins are indeed orthologous between these two organisms.

\section{Concluding remarks}

RBPs are key players in gene expression regulation in all organisms. They allow the cells to change their expression profile very rapidly to respond to different types of stimuli. The fast response is particularly important in the case of unicellular organisms, such as trypanosomatids and yeast, that rapidly need to adapt to environmental changes to survive.

Despite the phylogenetic distance, in some cases, the function of a protein of interest is conserved. Scerevisiae is

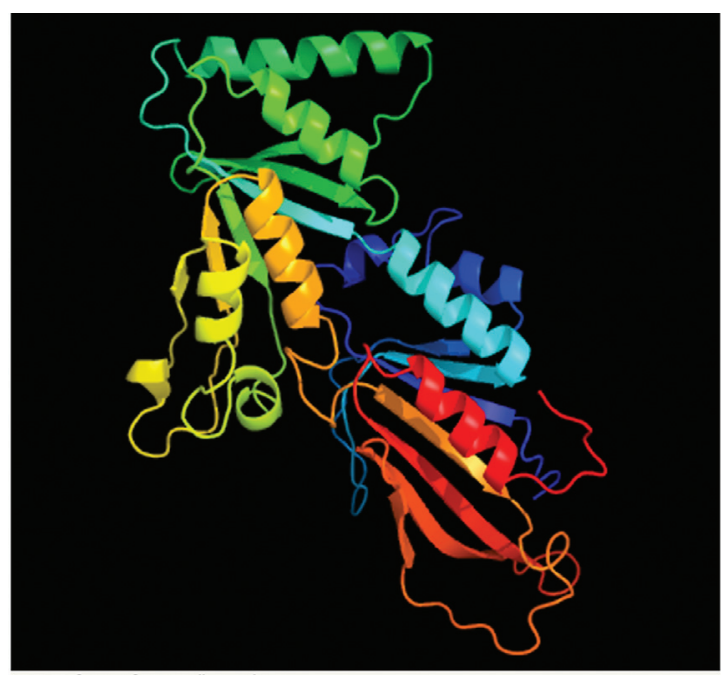

PDB header:cell cycle

Chain: B: PDB Molecule:defective in germ line development protein 3, isoform a; PDBTitle: kh domains

\begin{tabular}{l|c} 
Confidence: $\quad 100.0 \%$ & Confidence and coverage \\
278 residues ( $23 \%$ of your sequence) have been modelled \\
with $100.0 \%$ confidence by the single highest scoring template.
\end{tabular}

Figure 4 - Structural prediction of ScScp160 protein (Phyre2 program). 
Table 2 - Orthology analysis of RNA-binding proteins between T. cruzi and S. cerevisiae.

\begin{tabular}{|c|c|c|c|c|c|c|c|c|}
\hline $\begin{array}{l}\text { Trypanosoma cruzi } \\
\text { id }(\mathrm{Tc})\end{array}$ & Domains & $\begin{array}{l}\text { Saccharomyces } \\
\text { cerevisiae id }(\mathrm{Sc})\end{array}$ & Domains & $\begin{array}{c}\mathrm{Tc} \\
\text { length }\end{array}$ & $\begin{array}{c}\mathrm{Sc} \\
\text { length }\end{array}$ & $\begin{array}{c}\text { Tc } \\
\text { qcovhsp }\end{array}$ & $\begin{array}{c}\mathrm{Sc} \\
\text { qcovhsp }\end{array}$ & pident \\
\hline TcCLB.506885.70 & RRM & sp|P04147|PABP & RRM & 570 & 577 & 94 & 92 & 38.52 \\
\hline TcCLB.511741.40 & RRM & sp|P40561|SGN1 & RRM & 231 & 250 & 39 & 35 & 43.33 \\
\hline TcCLB.504431.90 & RRM & $\mathrm{sp}|\mathrm{P} 40567| \mathrm{MSL} 1$ & RRM & 114 & 111 & 76 & 76 & 32.58 \\
\hline TcCLB.503577.20 & Zinc Finger & sp|Q06102|YTH1 & Zinc Finger & 233 & 208 & 11 & 12 & 44 \\
\hline TcCLB.504071.80 & RRM & $\mathrm{sp}|\mathrm{Q} 08920| \mathrm{NCBP} 2$ & RRM & 188 & 208 & 60 & 68 & 40.85 \\
\hline TcCLB.511303.60 & $\begin{array}{l}\text { Eukaryotic transla- } \\
\text { tion initiation factor } \\
\text { eIF2A / RRM }\end{array}$ & sp|P06103|EIF3B & $\begin{array}{l}\text { Eukaryotic transla- } \\
\text { tion initiation factor } \\
\text { eIF2A / RRM }\end{array}$ & 696 & 763 & 90 & 83 & 22.17 \\
\hline TcCLB.511367.60 & $\mathrm{La} / \mathrm{RRM}$ & sp|P33399|LHP1 & $\mathrm{La} / \mathrm{RRM}$ & 333 & 275 & 56 & 73 & 33.18 \\
\hline TcCLB.508299.89 & RRM & sp|P53743|ESF2 & RRM & 238 & 316 & 44 & 34 & 32.41 \\
\hline TcCLB.511863.20 & RRM & $\mathrm{sp}|\mathrm{P} 32605| \mathrm{RU} 1 \mathrm{~A}$ & RRM & 371 & 298 & 27 & 33 & 27.72 \\
\hline TcCLB.507037.20 & RRM & sp|P37838|NOP4 & RRM & 486 & 685 & 70 & 53 & 27.59 \\
\hline TcCLB.507515.60 & RRM & sp|P40565|IST3 & RRM & 156 & 148 & 40 & 43 & 44.44 \\
\hline TcCLB.504045.114 & $\begin{array}{c}\text { Tryptophan } \\
\text { synthase alpha chain } \\
\text { / RRM }\end{array}$ & sp|P00931|TRP & $\begin{array}{l}\text { Tryptophan } \\
\text { synthase alpha } \\
\text { chain / RRM }\end{array}$ & 185 & 707 & 54 & 14 & 25.23 \\
\hline TcCLB.510657.160 & RRM & sp|Q08208|NOP12 & RRM & 421 & 459 & 50 & 48 & 24.68 \\
\hline TcCLB.503897.90 & RRM & sp|Q06106|MRD1 & RRM & 878 & 887 & 99 & 77 & 27.14 \\
\hline TcCLB.504157.10 & RRM & sp|P34167|IF4B & RRM & 450 & 436 & 20 & 21 & 34.38 \\
\hline TcCLB.508409.80 & MIF4G / RRM & sp|P39935|IF4F1 & MIF4G / RRM & 501 & 952 & 27 & 11 & 25.66 \\
\hline TcCLB.506693.30 & RRM & sp|Q00539|NAM8 & RRM & 243 & 523 & 30 & 17 & 38.64 \\
\hline TcCLB.511127.10 & RRM & sp|Q00916|RU17 & RRM & 240 & 300 & 33 & 26 & 33.75 \\
\hline TcCLB.508567.100 & $\begin{array}{l}\text { Adaptin } \mathrm{N} \text { terminal } \\
\text { region / RRM }\end{array}$ & sp|P38065|AP2A & $\begin{array}{l}\text { Adaptin } N \text { terminal } \\
\text { region / RRM }\end{array}$ & 1405 & 1025 & 11 & 14 & 24.38 \\
\hline TcCLB.511867.180 & PUB & sp|P32900|SKG6 & $\begin{array}{l}\text { Transmembrane } \\
\text { alpha-helix domain }\end{array}$ & 365 & 734 & 37 & 16 & 23.53 \\
\hline
\end{tabular}

a powerful biological model because it is a simple eukaryote whose genome is easily manipulated and, therefore, can be used to obtain hints about the function of genes in another organism (Table 2). For example, the T. cruzi TcJ6 protein is a homologue of the Sis1 protein from $S$. cerevisiae, and these proteins are involved in translation initiation in both organisms (Salmon et al., 2001). For instance, Mantilla et al. (2015) used $S$. cerevisiae to complement mutants for the T. cruzi protein TcP5CDH to study the proline metabolic pathway of the parasite.

The study of RBPs proteins and their function in unicellular eukaryotes should pave the way to enlighten the regulatory role of these proteins in higher eukaryotes.

\section{References}

Abbasi N, Park YI and Choi SB (2011) Pumilio Puf domain RNA-binding proteins in Arabidopsis. Plant Signal Behav 6:364-368.

Afroz T, Cienikova Z, Cléry A and Allain FH (2015) One, two, three, four! How multiple RRMs read the genome sequence. Methods Enzymol 558:235-278.

Alves LR, Avila AR, Correa A, Holetz FC Mansur FC, Manque PA, de Menezes JP, Buck GA, Krieger MA and Goldenberg
S (2010) Proteomic analysis reveals the dynamic association of proteins with translated mRNAs in Trypanosoma cruzi. Gene 452:72-78.

Alves LR, Oliveira C, Mörking PA, Kessler RL, Martins ST, Romagnoli BA, Marchini FK and Goldenberg S (2014) The mRNAs associated to a zinc finger protein from Trypanosoma cruzi shift during stress conditions. RNA Biol 11:921-933.

Alves LR and Goldenberg S (2016) RNA-binding proteins related to stress response and differentiation in protozoa. World $\mathrm{J}$ Biol Chem 7:78-87.

Amrani N, Minet M, Le Gouar M, Lacroute H and Wyers F (1997) Yeast Pab1 interacts with RNA15 and participates in the control of the poly(A) tail length in vitro. Mol Cell Biol 17:3694-3701.

Anderson JT, Paddy MR and Swanson MS (1993) PUB1 is a major nuclear and cytoplasmic polyadenylated RNA-binding protein in Saccharomyces cerevisiae. Mol Cell Biol 13:6102-6113.

Banerjee S and Barraud P (2014) Functions of double-stranded RNA-binding domains in nucleocytoplasmic transport. RNA Biol 11:1226-1232.

Batista JA, Teixeira SM, Donelson JE, Kirchhoff LV and de Sá CM (1994) Characterization of a Trypanosoma cruzi 
poly(A)-binding protein and its genes. Mol Biochem Parasitol 67:301-312.

Clayton C and Shapira M (2007) Post-transcriptional regulation of gene expression in trypanosomes and leishmanias. Mol Biochem Parasitol 156:93-101.

Cléry A and Allain F (2012) From Structure to Function of RNA Binding Domains. In: Madame Curie Bioscience Database [Internet]. Landes Bioscience, Austin, p. 2000-2013.

Corley SM and Gready JE (2008) Identification of the RGG box motif in Shadoo: RNA-binding and signaling roles? Bioinform Biol Insights 2:383-400.

D'Orso I and Frasch AC (2002) TcUBP-1, an mRNA destabilizing factor from trypanosomes, homodimerizes and interacts with novel AU-rich element- and Poly(A)-binding proteins forming a ribonucleoprotein complex. J Biol Chem 277:50520-50528.

Dallagiovanna B, Correa A, Probst CM, Holetz F, Smircich P, de Aguiar AM, Mansur F, da Silva CV, Mortara RA, Garat G, et al. (2008) Functional genomic characterization of mRNAs associated with TcPUF6, a pumilio-like protein from Trypanosoma cruzi. J Biol Chem 283:8266-8273.

De Gaudenzi J, Frasch AC and Clayton C (2005) RNA-binding domain proteins in Kinetoplastids: A comparative analysis. Eukaryot Cell 4:2106-2114.

De Gaudenzi JG, Jäger AV, Izcovich R and Campo VAO (2016) Insights into the regulation of mRNA processing of polycistronic transcripts mediated by DRBD4/PTB2, a trypanosome homolog of the polypyrimidine tract-binding protein. J Eukaryot Microbiol 63440-452.

De Souza W (2002) Basic cell biology of Trypanosoma cruzi. Curr Pharm Des 8:269-285.

Edwards TA (2015) Bespoke RNA recognition by Pumilios. Biochem Soc Trans 43:801-806.

Estruch F and Carlson M (1993) Two homologous zinc finger genes identified by multicopy suppression in a SNF1 protein kinase mutant of Saccharomyces cerevisiae. Mol Cell Biol 13:3872-3881.

Finn RD, Mistry J, Tate J, Coggill P, Heger A, Pollington JE, Gavin OL, Gunasekaran P, Ceric G, Forslund K, et al. (2010) The Pfam protein families database. Nucleic Acids Res 38:D211-222.

Garcia-Silva MR, Sanguinetti J, Cabrera-Cabrera F, Franzén O and Cayota A (2014) A particular set of small non-coding RNAs is bound to the distinctive Argonaute protein of Trypanosoma cruzi: insights from RNA-interference deficient organisms. Gene 538:379-84.

Grishin NV (2001) KH domain: one motif, two folds. Nucleic Acids Res 29:638-643.

Gu W, Deng Y, Zenklusen D and Singer RH (2004) A new yeast PUF family protein, Puf6p, represses ASH1 mRNA translation and is required for its localization. Genes Dev 18:1452-1465.

Guerra-Slompo EP, Probst CM, Pavoni DP, Goldenberg S, Krieger MA and Dallagiovanna B (2012) Molecular characterization of the Trypanosoma cruzi specific RNA binding protein TcRBP40 and its associated mRNAs. Biochem Biophys Res Commun 420:302-307.

Hall TM (2005) Multiple modes of RNA recognition by zinc finger proteins. Curr Opin Struct Biol 15:367-373.

Holetz FB, Alves LR, Probst CM, Dallagiovanna B, Marchini FK, Manque P, Buck G, Krieger MA, Correa A and Goldenberg
S (2010) Protein and mRNA content of TcDHH1-containing mRNPs in Trypanosoma cruzi. FEBS J 277:3415-3426.

Hutvagner G and Simard MJ (2008) Argonaute proteins: key players in RNA silencing. Nat Rev Mol Cell Biol 9:22-32.

Kishore S, Luber S and Zavolan M (2010) Deciphering the role of RNA-binding proteins in the post-transcriptional control of gene expression. Brief Funct Genomics 9:391-404.

Kramer S, Queiroz R, Ellis L, Hoheisel JD, Clayton C and Carrington M (2010) The RNA helicase DHH1 is central to the correct expression of many developmentally regulated mRNAs in trypanosomes. J Cell Sci 123:699-711.

Kramer S, Bannerman-Chukualim B, Ellis L, Boulden EA, Kelly S, Field MC and Carrington M (2013) Differential localization of the two T. brucei poly(A) binding proteins to the nucleus and RNP granulessuggests binding to distinct mRNA pools. PLoS One 8:e54004.

Lang BD and Fridovich-Keil JL (2000) Scp160p, a multiple $\mathrm{KH}$-domain protein, is a component of mRNP complexes in yeast. Nucleic Acids Res 28:1576-1584.

Liu J, Carmell MA, Rivas FV, Marsden CG, Thomson JM, Song JJ, Hammond SM, Joshua-Tor L and Hannon GJ (2004) Argonaute2 is the catalytic engine of mammalian RNAi. Science 305:1437-1441.

Lunde BM, Moore C and Varani G (2007) RNA-binding proteins: modular design for efficient function. Nat Rev Mol Cell Biol 8:479-490.

Mantilla BS, Paes LS, Pral EM, Martil DE, Thiemann OH, Fernández-Silva P, Bastos EL and Silber AM (2015) Role of $\Delta$ 1-pyrroline-5-carboxylate dehydrogenase supports mitochondrial metabolism and host-cell invasion of Trypanosoma cruzi. J Biol Chem 290:7767-7790.

Mari C, Dominguez C and Allain FH (2005) The RNA recognition motif, a plastic RNA-binding platform to regulate posttranscriptional gene expression. FEBS J 272:2118-2131.

Martínez-Pastor MT, Marchler G, Schüller C, Marchler-Bauer A, Ruis H and Estruch F (1996) The Saccharomyces cerevisiae zinc finger proteins Msn2p and Msn4p are required for transcriptional induction through the stress response element (STRE). EMBO J 15:2227-2235.

Mitchell SF, Jain S, She M and Parker R (2013) Global analysis of yeast mRNPs. Nat Struct Mol Biol 20:127-133.

Mörking PA, Dallagiovanna BM, Foti L, Garat B, Picchi GF, Umaki AC, Probst CM, Krieger MA, Goldenberg S and Fragoso SP (2004) TcZFP1: a CCCH zinc finger protein of Trypanosoma cruzi that binds poly-C oligoribonucleotides in vitro. Biochem Biophys Res Commun 319:169-177.

Mörking PA, Rampazzo RC, Walrad P, Probst CM, Soares MJ, Gradia DF, Pavoni DP, Krieger MA, Matthews K, Goldenberg S, et al. (2012) The zinc finger protein TcZFP2 binds target mRNAs enriched during Trypanosoma cruzi metacyclogenesis. Mem Inst Oswaldo Cruz 107:790-799.

Nakayashiki H, Kadotani N and Mayama S (2006) Evolution and diversification of RNA silencing proteins in fungi. $\mathrm{J}$ Mol Evol 63:127-135.

Názer E, Verdún RE and Sánchez DO (2011) Nucleolar localization of RNA binding proteins induced by actinomycin $\mathrm{D}$ and heat shock in Trypanosoma cruzi. PLoS One 6:e19920.

Olivas W and Parker R (2000) The Puf3 protein is a transcriptspecific regulator of mRNA degradation in yeast. EMBO J 19:6602-6611. 
Pérez-Díaz L, Correa A, Moretão MP, Goldenberg S, Dallagiovanna B and Garat B (2012) The overexpression of the trypanosomatid-exclusive TcRBP19 RNA-binding protein affects cellular infection by Trypanosoma cruzi. Mem Inst Oswaldo Cruz 107:1076-1079.

Pérez-Díaz L, Pastro L, Smircich P, Dallagiovanna B and Garat B (2013) Evidence for a negative feedback control mediated by the 3' untranslated region assuring the low expression level of the RNA binding protein TcRBP19 in T. cruzi epimastigotes. Biochem Biophys Res Commun 436:295299.

Ruiz-Echevarría MJ and Peltz SW (2000) The RNA binding protein Pub1 modulates the stability of transcripts containing upstream open reading frames. Cell 101:741-751.

Salmon D, Montero-Lomeli M and Goldenberg S (2001) A DnaJ-like protein homologous to the yeast co-chaperone Sis1 (TcJ6p) is involved in initiation of translation in Trypanosoma cruzi. J Biol Chem 276:43970-43979.

Shannon KW and Guthrie C (1991) Suppressors of a U4 snRNA mutation define a novel U6 snRNP protein with RNAbinding motifs. Genes Dev 5:773-785.

Tadauchi T, Matsumoto K, Herskowitz I and Irie K (2001) Posttranscriptional regulation through the HO 3'-UTR by Mpt5, a yeast homolog of Pumilio and FBF. EMBO J 20:552-561.
Thompson MJ, Lai WS, Taylor GS and Blackshear PJ (1996.) Cloning and characterization of two yeast genes encoding members of the $\mathrm{CCCH}$ class of zinc finger proteins: zinc finger-mediated impairment of cell growth. Gene 174:225-233.

Ulbricht RJ and Olivas WM (2008) Puflp acts in combination with other yeast Puf proteins to control mRNA stability. RNA 14:246-262.

Valverde R, Edwards L and Regan L (2008) Structure and function of KH domains. FEBS J 275:2712-2726.

Vidaver RM, Fortner DM, Loos-Austin LS and Brow DA (1999) Multiple functions of Saccharomyces cerevisiae splicing protein Prp24 in U6 RNA structural rearrangements. Genetics 153:1205-1218.

Vincent A (1986) TFIIIA and homologous genes. The 'finger' proteins. Nucleic Acids Res 14:4385-4391.

Weidner J, Wang C, Prescianotto-Baschong C, Estrada AF and Spang A (2014) The polysome-associated proteins Scp160 and Bfr1 prevent $\mathrm{P}$ body formation under normal growth conditions. J Cell Sci 127:1992-2004.

Yan KS, Yan S, Farooq A, Han A, Zeng L and Zhou MM (2003) Structure and conserved RNA binding of the PAZ domain. Nature 426:468-474.

Associate Editor: Angela M. Vianna-Morgante

License information: This is an open-access article distributed under the terms of the Creative Commons Attribution License (type CC-BY), which permits unrestricted use, distribution and reproduction in any medium, provided the original article is properly cited. 